\title{
Desesperanza aprendida: categoría emergente de los docentes frente a la inclusión educativa ${ }^{1}$
}

\author{
Vilma Roció Flórez Muñoz ${ }^{2}$, Rocio del Pilar González Salamanca ${ }^{3}$ \\ GLORIA ISAZA DE GIL ${ }^{4}$
}

\begin{abstract}
Resumen
El presente artículo emerge de la reflexión realizada en la investigación: Desesperanza Aprendida: Categoría Emergente con respecto a las percepciones y las actitudes de los docentes frente a la inclusión de estudiantes con Necesidades Educativas Especiales (NEE) de las Instituciones Carlos Mario Simmonds y Cristo Rey de la ciudad de Popayán, realizada a partir de marzo del 2011 hasta el presente año.

El análisis de los lineamientos educativos de orden mundial, promulgados por la UNESCO, en la XV cumbre Iberoamericana de Salamanca, (España) del 10 de junio de 1994, y la reflexión del deber ser del quehacer pedagógico orientada en los diferentes seminarios correspondiente a la maestría "Educación desde la Diversidad" impartida por la Universidad de Manizales, fueron unánimes en plantear el respeto por la diversidad y la necesidad de transformación de las prácticas pedagógicas, pero también de las prácticas familiares y sociales para el bienestar de todos.

Metodológicamente la investigación recurrió a la teoría fundada, cuya observación no participante se llevó acabo en dos establecimientos Educativos de la ciudad de Popayán, en las cuales se desarrollaron entrevistas semiestructuradas, diálogos informales y la experiencia de convivencia en el medio por parte de las investigadoras. Es de anotar que el análisis de la información desde la realidad, teoría y práctica, configuró las entrevistas, diálogos y observaciones como testimonio. De otro lado las teorías que soportan el estudio del tema de investigación se unieron como argumentos orientadores de la lectura de la realidad, a su vez el saber práctico adquirió el status de saber pedagógico, pues orientó el trabajo desde la desesperanza aprendida.
\end{abstract}

1 Recibido: 28 de octubre de 2014. Aceptado: 05 de julio de 2015.

2 Vilma Roció Flórez Muñoz. Magister en Educación desde la Diversidad; Licenciada en Educación Especial. Profesional de Apoyo de la Unidad de Atención Integral, Popayán. Correo Electrónico: vilmarofm@hotmail. com

3 Rocio del Pilar González Salamanca. Magister en Educación desde la Diversidad; Psicóloga. Universidad Cooperativa de Colombia, Seccional Popayán, 2009. Psicóloga Educativa de la Institución Educativa Cristo Rey, Popayán. Correo Electrónico: pilar-gonzalez1@hotmail.com

4 El presente artículo es resultado de investigación desde marzo del 2011 a la fecha, del macroproyecto: Concepciones y prácticas pedagógicas de los maestros de jóvenes en situación de vulnerabilidad institucional, cultural, ambiental y/o de entorno de las instituciones educativas de Nariño y Cauca, cuya investigadora principal es la Mg. Gloria Isaza de Gil y se realizó como prerrequisito para obtener el título de Magister en Educación desde la Diversidad. Correo electrónico: gloriai@umanizales.edu.co 
El resultado de este trabajo, es intangible por cuanto es preciso ahondar en la toma de compromiso para cambiar la realidad y su efectivo desarrollo, un cambio claro, necesario y significativo es este rol del docente, pues se necesita ver el mundo, su contexto y la sociedad con otros ojos, y al niño, niña o joven como centro de todo ello. Mirar al estudiante como sujeto de derechos y deberes, potencial, capaz, con habilidades y destrezas, sin señalamiento alguno son actitudes claves para desempeñar adecuadamente la labor educativa.

Palabras clave: Diversidad, Vulnerabilidad, Inclusión educativa, Estudiantes con NEE, Desmotivación, Desesperanza aprendida.

\section{Abstract \\ Learned Hopelessness: \\ Teacher's Emerging Category towards the Inclusive Education}

This Article emerges from the reflection made in the Investigation. Learned Hopelessness: Emerging Category regarding the perceptions and attitudes of teachers towards the inclusion of students with Special Educational Needs (SEN) of the Institutions Carlos Mario Simmonds and Cristo Rey of Popayan City made since March of 20112013.

The analysis of the educational guidelines of world order, enacted by the UNESCO, in the XV Iberoamerican Summit of Salamanca, (Spain) in the 10th of June of 1994, and the reflection of the pedagogical should be, oriented in different seminars relevant to mastery "Education through diversity" imparted for University of Manizales, were unanimous in raising respect for diversity and the need for transformation of teaching practices, but also family and social practices for the welfare of all.

Methodologically the research drew on Found Theory, which non- participant observation was held in two educational establishments in Popayan city, which were developed in semi-structured interviews, informal discussions and the experience of living in the environment by the researchers. It should be noted that the analysis of the information from reality, theory and practice, set up interviews, discussions and observations as evidence. On the other hand the theories that support the study of the research topic joined as arguments counselors in the reading of reality, so the practical knowledge acquired the status of pedagogical knowledge, because it oriented the work from the learned hopelessness.

The outcome of this work is intangible, because is precise to delve in the commitment to change the reality and its effective development, a clear change, that is necessary and significant in the teachers role, as they need to see the world, its context and society with new eyes, and the child or young people as the center of it all. Seeing the student as a subject of rights and duties, potential, capable, with skills and abilities, without signaling any attitudes are the key to adequately perform educational work.

Keywords: Diversity, Vulnerability, inclusive education, Students with SEN, Discouragement, Learned Hopelessness. 


\section{Presentación}

En las Instituciones de Educación Básica Primaria, de carácter oficial, Carlos Mario Simmonds y Cristo Rey, del Departamento del Cauca, municipio de Popayán, a partir de marzo del 2011 hasta el 2013, la docente Vilma Rocío Flórez Muñoz, Licenciada en Educación Especial y la Psicóloga Rocío del Pilar González Salamanca, llevaron a cabo la investigación denominada "Desesperanza Aprendida: Categoría Emergente: de los docentes frente a la inclusión educativa".

Quienes fundamentándose de que la historia humana marcha indefectiblemente hacia el progreso y hacia la perfectibilidad del desarrollo humano. Es un hecho que la rapidez de los cambios sociales, económicos, culturales y tecnológicos plantean nuevas exigencias que obligan a los sistemas educativos a una renovación constate para dar respuesta a las demandas y necesidades de las personas y por consiguiente de la sociedad. En este marco, las innovaciones educativas se presentan como un espacio crucial para anticipar respuestas a nuevos desafíos y generar alternativas de solución como es el caso de la educación inclusiva que es un proceso por medio del cual se responde con calidad, equidad y pertinencia a la población diversa, es decir brindar una educación que satisfaga las necesidades particulares de los estudiantes.

Sin embargo, el anterior enunciado ha sido objeto de cuestionamiento ante la sociedad y ni que decir por parte del gremio educativo pues se evidencia inconformidad y desmotivación frente a los resultados requeridos por el MEN como lo podemos ejemplarizar en el desarrollo del presente articulo.

\section{Justificación}

La investigación se llevó a cabo debido a que la acelerada transformación del mundo ha forzado y de algún modo afectado al sujeto en su parte cognitiva, emocional y comportamental, desde este contexto, los inconvenientes que tienen los docentes para la atención con calidad a la población diversa, no ha sido la excepción, es decir que los docentes son considerados sujetos sociales, participes activos en la construcción del mundo contemporáneo y por consiguiente, actores principales en la dignificación humana.

El reconocimiento de la educación como un derecho humano trae consigo no solo el imperativo de la democratización, del acceso para todas las personas sin distingo alguno, sino también que sea de óptima calidad y con la posibilidad de permanencia dentro del sistema en condiciones dignas, de equidad, acorde a su realidad cultural, social y personal.

Desarrollar procesos de inclusión con calidad, es un reto de la educación contemporánea, para ello, se requiere de los docentes en particular, una transformación profunda de las representaciones mentales y sociales, que se traducen necesariamente en cambios de actitudes y acciones que repercuten en el sistema educativo.

De acuerdo a la Constitución Política de Colombia (1991) en el titulo II, de los derechos, las garantías y los deberes del artículo 67, decreta la educación como derecho fundamental, así como también se generan planteamientos hacia una educación para todos, cuyo objetivo primordial es eliminar las diversas formas de opresión y desigualdad, constituyendo asideros para hablar de calidad educativa, calidad de vida y construcción de sociedades justas y solidarias.

El logro de la equidad y la justicia social es uno de los mayores desafíos a los que se enfrentan hoy en día gran parte de los sistemas educativos. El derecho de todos a recibir una educación de calidad en igualdad de condiciones, es un hecho de justicia social y un derecho inalienable en las sociedades democráticas. Sin embargo, alcanzar estos ideales requiere nuevos planteamientos y formas de entender la educación. Es en este punto donde se ve 
la inclusión como el medio más adecuado para ofrecer a todos los niños, niñas y jóvenes, las mismas oportunidades para aprender y participar plenamente en la sociedad. Cada contexto, cada momento marca la impronta de trazar rutas nuevas, buscar nuevos aliados e inspirar y motivar a las comunidades para obtener dichas metas.

Por tanto, se realiza esta propuesta investigativa que no solo es importante por su finalidad o por la población a la cual esta dirigida, sino también para lograr un cambio actitudinal en los docentes, teniendo en cuenta que muchos de ellos, han creado una sensación de impotencia ante su quehacer pedagógico y el tema de la inclusión educativa. Por consiguiente conduciendo o condenando a sus estudiantes al fracaso, lo cual conduce a una categoría emergente denominada "Desesperanza Aprendida", en la cual intervienen tres componentes; cognitivo, motivacional y emocional, que se ven reflejados en su "deber ser" como profesionales de la educación.

\section{Problema}

A finales del siglo XX y a inicios de este, hacer valer el derecho a la educación para todos los niños, niñas y jóvenes con $\mathrm{Ne}$ cesidades Educativas Especiales (NEE), asociadas a discapacidad u otros factores es una aspiración para muchos, un compromiso para algunos y una realidad para unos cuantos. La concepción de la persona con Necesidades Educativas se plantea en la actualidad como una alternativa efectiva tanto para la comprensión del desempeño en el aprendizaje en todos los estudiantes, como para la oferta de servicios de carácter educativo y social, lo cual contribuye de manera significativa a la construcción de una cultura de atención a la diversidad.

La Conferencia Mundial sobre Necesidades Educativas Especiales en 1994, organizada por la UNESCO, en la XV cumbre Iberoamericana de Salamanca, (España) del 10 de junio de 1994, con la participación de 92 países preocupados por mejorar la calidad educativa y conceder participación activa a grupos minoritarios que han sido objeto de exclusión del servicio educativo, invitó a la transformación de las relaciones entre la escuela y la comunidad, la cual se establece con claridad en el punto 3, que:

"Las escuelas deben acoger a todos los niños, independientemente de sus condiciones físicas, intelectuales, sociales, emocionales, lingüísticas u otras. Deben acoger a niños discapacitados y niños bien dotados, a niños que viven en la calle y que trabajan, niños de poblaciones remotas o nómada, niños de minorías lingüísticas, étnicas o culturales y niños de otros grupos o zonas desfavorecidas o marginadas". (Declaración de Salamanca, 1994, p. 61).

En este mismo año en Colombia, es promulgada la Ley General de Educación, en cuyo Título III se estableció "Las modalidades de atención educativa a poblaciones", incluyendo en el capitulo I la educación para personas con limitaciones y capacidades excepcionales, y en su Articulo 46, la integración de los educandos con NEE en el aula. Posteriormente en 1996, se inició la reflexión "Hacia una educación con equidad", mediante el Decreto 2082, que tuvo como marco de acción la conferencia mundial ya citada sobre $\mathrm{Ne}$ cesidades Educativas Especiales.

A nivel Departamental, el documento "Discapacidad, minusvalía y deficiencia; Alertas y procesos en el Departamento del Cauca", (Gobernación del Cauca. Dirección Departamental de Salud, Despacho de la primera dama y Red de iniciativas de personas discapacitadas Comunitar, 1999 , p. 7). Deja conocer que en 1999, se pretendía sensibilizar a la sociedad caucana, a fin de lograr la incorporación de las personas con alguna discapacidad.

Más tarde en el año 2000, los diversos cambios administrativos de la educa- 
ción, entre ellos, la municipalización de la educación, diezmó el grupo de educación especial del departamento del Cauca, dando origen a la conformación de la unidad de atención integral (UAI) y su equipo multidisciplinario, para la atención de la población del municipio de Popayán.

Actualmente el decreto 366 del 2009, emanado del MEN, "reglamenta la organización del servicio de apoyo pedagógico para la atención de los estudiantes con discapacidad y con capacidades o con talentos excepcionales en el marco de la educación inclusiva". Este requerimiento de educación inclusiva, en particular, ha impactado al sistema educativo estableciendo una mirada abierta de la educación y su relación con el sujeto de la misma. Esta realidad conlleva al surgimiento de nuevos requerimientos y prácticas de gestión para el docente, con el fin de responder de forma eficiente y efectiva a los cambios que se experimentan día a día en el quehacer pedagógico como docentes de los niveles de educación pre-escolar, básica y superior. Por esta razón se desarrolla la concepción del docente como un Gestor de las transformaciones sociales, el cual, tiene como misión buscar que la educación esté centrada en la alteridad, los valores humanos y sociales.

Con el fin de indagar sobre las percepciones y las actitudes de los docentes en la educación básica primaria, se acudió al método de Investigación de la teoría fundada, cuya observación no participante se llevó acabo en dos establecimientos educativo de la ciudad de Popayán (Institución Educativa Carlos Mario Simmonds e Institución Educativa Cristo Rey) facilitando una mirada argumentada respecto a las actitudes y percepciones de los docentes de educación básica primaria, en el proceso de atención a la diversidad y su manifestación en la inclusión de estudiantes que presentan Necesidades Educativas Especiales, mediante la codificación abierta y axial estructurada en el cuadro categorial de las entrevistas realizadas a los docentes. Dicho recurso proporcionó mayor cantidad y calidad de información, además de posibilitar el análisis de conductas verbales y no verbales, como también contenidos de discurso. (Glaser y Strauss, 1967).

\section{Pregunta}

Lo anterior, nos conlleva a preguntarnos: ¿Cuáles son las actitudes y percepciones de los docentes frente a la inclusión de estudiantes con Necesidades Educativas Especiales (NEE) en las Instituciones de Educación básica primaria, de carácter oficial, Carlos Mario Simmonds y Cristo Rey, del departamento del Cauca, municipio de Popayán, año escolar 2012?

\section{Antecedentes}

Entre algunas de las investigaciones acerca de las percepciones y actitudes de los docentes frente a la inclusión educativa, con énfasis en la atención a la población diversa, por sus aportes significativos están: en Argentina, la tesis de la Licenciatura en Gestión Educativa, de la Universidad CAECE denominada: "tolerancia y nodiscriminación en escuelas inclusivas", cuyas autoras son Silvia Andrea Zaballa y Bahía Blanca (2001). La tesis es realizada por estas personas quienes han estado durante 12 años como coautoras y participantes activas de las escuelas inclusivas, en cuya experiencia, nació un año antes de la Declaración de Salamanca, en una ciudad al sur de la provincia de Buenos Aires, elaborada por docentes que sin más sustento teórico que el concebir que la educación era un derecho para todos, comenzaron a trabajar en el aula con estudiantes en condiciones sociales, situaciones familiares y capacidades diversas. Docentes que desconocían conceptualmente que era la integración, la inclusión, la normalización. Pero concebían el acto de educar, "...como una puesta a disposición del otro de todo aquello que le posibilite ser distinto de lo que es en algún aspecto" y que bregaban por una educación para 
todos, enfatizando en el trayecto y no en resultados excluyentes.

A nivel Nacional se retoma el trabajo de la Universidad de Antioquia que desarrolla el Dr. Alexander Yarza de los Ríos, denominado Educadores Especiales en la Educación Inclusiva como reforma y práctica de gubernamentalizada en Colombia. (2011, p. 34). Esta tesis plantea la textualidad de la educación inclusiva como reforma, el cual produce nuevos etiquetamientos y funcionalidades en los sujetos (propios), este trabajo postula que la educación inclusiva (en proceso de institucionalización en Colombia) hace énfasis en la importancia de entender que la inclusión significa ofertar el servicio escolar con calidad y equidad a cualquier sujeto educable, es decir, a todos y todas los estudiantes en tanto diversos; además, plantea el análisis en términos de barreras para la participación, el aprendizaje y de facilitadores para la inclusión de la población en situación de vulnerabilidad y/o diversidad.

En cuanto a referentes locales, se retoma el documento denominado: "discapacidad, minusvalía y deficiencia, de la gobernación del Cauca". Bajo la supervisión de la dirección departamental de salud, despacho de la primera dama y red de iniciativas de personas discapacitadas Comunitar, dicho articulo del año 1999, deja conocer la pretensión de sensibilizar a la sociedad Caucana, a fin de lograr la incorporación de las personas con alguna discapacidad.

\section{Objetivo}

El objetivo que se pretendió con esta investigación fue el de identificar cuáles son las actitudes y percepciones de los docentes frente a la inclusión de estudiantes con Necesidades Educativas Especiales (NEE), en las Instituciones de Educación Básica Primaria, de carácter oficial, Carlos Mario Simmonds y Cristo Rey, del departamento del Cauca, Municipio de Popayán, año escolar 2012.

\section{Metodología}

La presente investigación se realizó a través de la teoría fundada, la cual se describe como un modo de hacer análisis. De la Cuesta (1998) siguiendo a Strauss, considera que su objetivo es el de generar teoría a partir de textos recogidos en contextos naturales y sus hallazgos son formulaciones teóricas de la realidad. Según Sandoval (1997), la teoría fundada es una Metodología General para desarrollar teoría a partir de datos que son sistemáticamente capturados y analizados; es una forma de pensar acerca de los datos y poderlos conceptualizar. Aun cuando son muchos los puntos de afinidad en los que la teoría fundamentada se identifica con otras aproximaciones de investigación cualitativa, se diferencia de aquellas por su énfasis en la construcción de teoría.

El método de la teoría fundamentada es el de comparación constante, que connota, como dice Sandoval, una continua revisión y comparación de los datos capturados para ir construyendo teoría de la realidad. En términos de Velasco y Díaz de Rada (2000), el método de ésta tendencia ayuda a que el antropólogo elabore y compare nuevas categorías mentales e introduzca nociones de espacio y tiempo, de oposición y contradicción, que pueden ser extrañas al pensamiento tradicional.

Strauss (1970), define sus procedimientos básicos en: recogida de datos, codificación y reflexión analítica en notas. Para elaborar la teoría, es fundamental que se descubran, construyan y relacionen las categorías encontradas; estas constituyen el elemento conceptual de la teoría y muestran las relaciones entre ellas y los datos.

Los datos se recogen con base en el muestreo teórico, el cual, a decir Glasser y Strauss (1967), es el medio o sistema por el que el investigador decide con base analítica, que datos buscar y registrar. Por ello, la recogida de datos en la investigación etnográfica se debe guiar por una teoría de diseño emergente, pues estos 
escenarios y medios pueden ir cambiando en la medida que va apareciendo nueva información.

La teoría fundamentada, establece la distinción entre la teoría formal y la teoría sustantiva, haciendo énfasis en la teoría sustantiva, la cual, está relacionada con la interacción permanente que el investigador logra en el proceso de recolección de datos; de los cuales pueden ir surgiendo nuevas hipótesis para ser verificadas. En tal sentido, esta teoría es el resultado del procesamiento sistemático de los datos de campo (mediante procesos de codificación y categorización). Glasser y Strauss (1967).

\section{Diseño}

Los resultados de las entrevistas semiestructuradas, los diálogos informales y la experiencia de convivencia en el medio por parte de una de las investigadoras, evidenciaron las categorías conceptuales de: diversidad, vulnerabilidad e inclusión educativa, así como también, actitudes y sentimientos, eligiendo como eje de interés de trabajo, la categoría de percepciones y actitudes en la educación inclusiva para niños, niñas y jóvenes con NEE. Es de anotar que la información fundamentada desde la realidad, teoría y práctica, configuró las entrevistas, diálogos y observaciones como testimonio, de otro lado las teorías que soportan el estudio del tema de investigación, se urdieron como argumentos orientadores de la lectura de la realidad, a su vez el saber práctico adquirió el status de saber pedagógico, pues orientó el trabajo de análisis desde la desesperanza aprendida.

\section{Unidad de análisis y unidad de trabajo}

La unidad de análisis como proceso de valoración que lleva al investigador a concretizar la búsqueda investigativa y por ende se constituye en objeto de la codificación y/o de la categorización de la misma se focalizo en las actitudes y percepciones de los docentes frente a la inclusión de estudiantes que presentan Necesidades Educativas Especiales en dos instituciones de la ciudad de Popayán.

Como unidad de trabajo se nombraron a nueve docentes de Educación Básica Primaria de las Instituciones de carácter oficial "Carlos Mario Simmonds" y "Cristo Rey" de dicha ciudad de Popayán, las cuales tienen en común docentes que han tenido o tienen la oportunidad de brindar un proceso de enseñanza aprendizaje dentro de sus aulas a estudiantes en situación de discapacidad.

Construcción de sentido. ¿Qué saben los docentes sobre diversidad, vulnerabilidad, e inclusión? En cuanto a la diversidad...

La escuela se enfrenta al reto de llevar a la práctica el derecho de todos a la educación y fomentar la igualdad de oportunidades. Al respecto, Grisales, (2011, p.16) en su documento: el reconocimiento de la diversidad como valor y derecho afirma que la diversidad es el "reconocimiento de lo heterogéneo, la diferencia, la variedad, la desemejanza, la individualidad, la multiculturalidad y la interculturalidad. Se habla, entonces, de diferencias en cultura, étnicas y raciales, diferencias de género y diferencias lingüísticas, entre otras. Se refiere a la participación e igualdad como paso importante a la inclusión social".

La atención a la diversidad, en la mayoría de las instituciones, no se ha implementado, por cuanto supone una nueva forma de entender la realidad educativa. Sí bien es cierto que hay un porcentaje de docentes que cuentan con muchas ansias para mejorar su praxis cotidiana y vocación para ser transformadores de vida, hay también muchos en ejercicio que no han adquirido o se han negado a una formación pensada para la escuela del siglo XXI, y menos aún se ha considerado el hecho que la sociedad está siendo objeto de muchos cambios, donde sin duda alguna, la formación permanente resulta indispensable para el profesorado de todos los niveles educativos. 
Con la entrevista realizadas a los docentes de dichas Instituciones Educativas, se logró conocer que existen actitudes desfavorables por parte de algunos de ellos; actitudes que evidentemente atentan contra una verdadera formación de calidad, como por ejemplo: la falta de vocación o de sentido de pertenencia por su labor, docencia infestada de improvisación, con marcada naturaleza informativa más que formativa, carencia de alteridad y humor, aunado en muchos casos, a una imagen devaluada ante los demás y peor aún, una representación desmotivante ante sí mismo.

Las encuestas realizadas en el marco de esta investigación permitieron evidenciar que los docentes del nivel de educación básica primaria, no tienen un acercamiento conceptual claro con respecto a la definición de diversidad, en su defecto demostraron identificar los sujetos a los cuales hace referencia la diversidad. Esta realidad, por si misma, deja entrever que el cumplimiento de la política pública, está mediada por factores de edad, condiciones laborales y perspectivas del futuro.

Algunas de las respuestas de los maestros acerca de la diversidad, como en el caso de los docentes 1 y 2 "tratar a niños especiales" , "tratar a niños con problemas de aprendizaje", visibilizan la falta de actualización en las políticas educativas, así mismo las respuestas de los docentes 4 y 5: "hablando de seres humanos, decimos que la diversidad está enfocada a la conformación de grupos indígenas, campesinos, blancos, negros, desplazados, en fin... todos revueltos", "hace referencia a diferentes contextos de procedencia, diversidad también es la edad, competencias, lo que el individuo es capaz y no de hacer, en resumen, son las características de cada ser", muestran la tendencia a agrupar, es decir a ver la heterogeneidad como homogeneidad; principio adverso a la realidad

Como bien se puede apreciar, la preocupación del maestro no se centra en el sujeto que asiste al aula, al respecto, la Doctora Luz Helena Patiño (2011, p.18) expone en su eje temático de la atención a la diversidad en el contexto de aula de clase que:

"Los procesos de enseñanza aprendizaje deben favorecer la participación activa de los estudiantes y el énfasis no se debe poner en el aprendizaje memorístico de hechos o conceptos, sino en la creación de un entorno que estimule la construcción del propio conocimiento y elaborar su propio sentido (Bruner y Haste, 1990) y dentro del cual el profesorado pueda conducir al estudiante progresivamente hacia niveles superiores de independencia, autonomía y capacidad para aprender, en un contexto de colaboración y sentido comunitario que debe respaldar y acentuar siempre todas las apropiaciones".

Ante la realidad reflexionada, no es desatinado afirmar que el reconocimiento y el respeto por la diversidad humana es bastante fácil como práctica teórica, pero muy compleja como vivencia cotidiana, más aún sí es participe activo de la formación de estudiantes que están en situación de vulnerabilidad o que están en riesgos de exclusión, entre los que se encuentran las niñas, los niños y jóvenes en situación de calle, los desplazados, las personas con discapacidad, condiciones de pobreza extrema, estudiantes con capacidades y talentos excepcionales, población indígena, entre otros.

\section{En cuanto a la vulnerabilidad...}

De acuerdo a algunas de las respuestas referidas a la vulnerabilidad se identifica a que la vulnerabilidad es para los docentes 1,2 y 3 respectivamente: "descomposición de la sociedad a los más indefensos", "tiene que ver con las personas que de alguna manera, ya sea en salud, educación, alimentación están desprotegidas, carecen de muchas ayudas", "está relacionada con el daño físico o psicológico 
que se le puede causar a las personas". Estos enunciados evidencian que poseen vacíos conceptuales, que sintetizan esta temática y victimizan de alguna manera a las personas que se encuentran en condiciones de vulnerabilidad, percibiéndolas desde una mirada negativa, pero sin tener en cuenta sus potencialidades.

Por otra parte también se encuentra respuestas que tienen un acercamiento enfocado a la realidad en los docentes 5 y 6. Los cuales mencionan que "es algo que no está firme, algo que está sujeto a cambio, puede ser cambios de forma positiva o negativa, la vulnerabilidad no siempre va a permanecer ahí, en cualquier momento se puede cambiar", "es estar en estado de riesgo o de desigualdad al que está expuesto el ser humano, después de un acontecimiento que ha marcado su vida".

Según lo señalado por la Comisión Económica para América Latina (CEPAL, 2002), la vulnerabilidad es el resultado de la exposición a riesgos, además de la incapacidad para enfrentarlos y la inhabilidad para adaptarse activamente. La vulnerabilidad coloca a quien lo padece en una situación de desventaja del ejercicio pleno de sus derechos y libertades. Por tanto, es una dimensión de susceptibilidad al daño, condicionada por factores intrínsecos y extrínsecos, anclada en la radical fragilidad del ser humano, pero sin duda atribuible en buena medida a elementos sociales y ambientales, demostrando que la vulnerabilidad es una condición externa al ser humano, es una situación que se manifiesta a partir del contexto social la cual se deriva de la violación de los derechos humanos.

La población vulnerable esta referida a grupos o comunidades que por circunstancias de pobreza, origen étnico, estado de salud, edad, género o discapacidad, entre otros, se encuentran en una situación de mayor indefensión para ser frente a los problemas que plantea y exige el diario vivir y no cuentan con los recursos necesarios para satisfacer sus necesidades básicas. La vulnerabilidad coloca a quien lo padece en una situación de desventaja del ejercicio pleno de sus derechos y libertades.

Ciertamente, el énfasis puesto en lo relacional, lo contextual y lo procesual, permite considerar que la vulnerabilidad, aun siendo intrínseca al ser humano, no es una característica estable e inalterable, antes bien es dependiente, al menos en parte, de factores que pueden cambiarse, en los que se puede intervenir. De ahí que ésta sea la clave que sustenta la obligación moral de una acción, preventiva, curativa, social, económica, educativa o de cualquier otra índole, que pueda minimizar, o evitar estas condiciones favorables al daño.

\section{En cuanto a la educación inclusiva...}

A la pregunta relacionada con la Educación Inclusiva, los docentes 1 y 2 exponen que: "una educación especial para aquellos niños que tienen problemas de aprendizaje", "aquella que responde a las necesidades y características diferentes de los niños especiales", ante estas respuestas se evidencia que existe una falta de actualización normativa, así como también el uso de términos que ya no son utilizados para referirse a los estudiantes o personas que están en condición de discapacidad. Desconocen que la inclusión se encuentra en el corazón del proceso de innovación educativa, implicando a las políticas educativas para que mejore el aprendizaje y la participación de todos, esto a su vez conlleva a la reducción de su exclusión en la cultura, en los currículos y en la vida de la escuela.

Resulta evidente que este panorama educativo genera nuevas necesidades de formación en los docentes encargados de desarrollar prácticas educativas capaces de responder con garantías de éxito ante el desafío de una nueva educación para una nueva sociedad. Son numerosos los autores que coinciden en señalar que el 
profesorado es el agente clave para el cambio de cualquier sistema educativo y que de su actuación depende el éxito de todo programa de innovación educativa como en el caso de la educación inclusiva. De ahí que la formación de los docentes se convierta en un aspecto de especial atención para la atención a la diversidad.

En cuanto a la experiencia de los docentes, como orientadores de estudiantes que presentan NEE se percibe que han tenido la oportunidad de conocerlos y han sido o son parte de su formación, aunque esta experiencia en la mayoría de los casos no tuvo un impacto positivo o gratificante. Los docentes 4,5 y 6 expresaron lo siguiente: "considero que no hay que rechazarlos. Pero sí creo que deben de acudir a un aula especial". "Creo necesario que como docentes deberíamos contar con una buena capacitación para poder orientarlos y no rechazarlos". "Sí, me sentí mal, incapaz de dar la verdadera orientación que el estudiante necesitaba, no tenía la preparación requerida para atender tal necesidad y por tal motivo lo aislaba".

No obstante, es necesario aclarar que esta tendencia desfavorable hacia la inclusión no es sinónimo de fracaso, como en algunos casos lo han dado a entender los docentes, por el contrario estos resultados los podemos tomar como una posición a un cambio de actitud y mentalidad para fortalecer el proceso de inclusión.

De acuerdo a estas percepciones se deduce claramente que el profesorado inconscientemente esta actuando bajo los parámetros de la homogenización y que para atender y brindarles una educación de calidad es necesario tener herramientas metodológicas especiales para dicho manejo, ya que según ellos no cuentan con la preparación adecuada para su atención integral, por tanto se evidencia en sus respuestas actitudes de exclusión, por lo que se puede concluir que generalmente la muestra presenta una tendencia actitudinal adversa hacia la inclusión de los estudiantes que presentan N.E.E. en la educación básica primaria de las dos Instituciones Educativas ya mencionadas.

Siguiendo a Zemelman (1998, p. 20) el hombre puede progresar cuando manifiesta una inconformidad con lo dado y busca otros recorridos, por lo tanto expande su universo de pertenencia y agrega"... si queremos mantenernos vivos requerimos de una constante trascendencia en la reflexión teórica, respecto de cualquier fórmula que nos delimite acríticamente en una situación que se define como definitiva, por lo tanto carente de alternativas..." Aquí la inconformidad no tiene una connotación negativa, sino más bien es el inicio para acrecentar una postura crítica ante las dificultades presentadas.

Como se puede corroborar el hecho de que los docentes compartan durante toda una jornada escolar con estudiantes que presenta NEE, es ya para ellos, sinónimo de incomodidad, ya sea por sus imaginarios, la prevención dada por experiencias de colegas, impotencia al brindarle una orientación significativa, falta de actualización profesional, cansancio por tener más de 25 años laborando o simplemente porque se requiere más compromiso, trabajo, tiempo y sobretodo tolerancia. Todas las anteriores características, fue posible concebirlas desde una sola categoría que llamamos "Desesperanza aprendida".

\section{Recordando el "deber ser"}

"Una educación para la diversidad, necesita resignificar el espacio de la crítica desde la cultura y la historia. La cultura en esta perspectiva"...no se ve como monolítica o inmutable, sino como un lugar de fronteras múltiples y heterogéneas donde las diferentes historias, lenguajes, experiencias $y$ voces se entremezclan en medio de diversas relaciones de poder y privilegio. Dentro de esta tierra fronteriza cultural y pedagógica, conocida como escuela, las culturas 
subordinadas se empujan y penetran las supuestamente homogéneas y no problemáticas fronteras de las prácticas y formas culturales dominantes" (Giroux, 1992, p. 8).

Al respecto, el autor citado, es enfático en recalcar que los educadores críticos no se pueden quedar mostrando cómo las ideologías se insertan en las relaciones que se establecen en el sistema escolar (P.E.I., currículo, organización escolar, relaciones maestro-alumno), la pedagogía crítica necesita “...ir más allá analizando cómo son adoptadas en realidad las ideologías en las voces contradictorias y en la experiencias vividas de los estudiantes tal como ellos dan significado a los sueños, a los deseos y a las posiciones de sujeto que albergan" (Isaza, 2011, p. 12).

En una etapa de profundas transformaciones en la educación debido a los cambios sociales, somos conscientes que se presentan nuevos enfoques educativos, problemas en la toma de decisiones en los objetivos propuestos, en las metodologías que se emplean, en fin, la educación ha necesitado y necesita plantearse y replantearse su discurso, por cuanto, se concibe la necesidad que el educador tome una actitud reflexiva de la problemática educativa de nuestro tiempo.

"La formación del profesorado se constituye en uno de los principales factores dinamizadores y cualificadores de los sistemas educativos. Se dice a menudo que los docentes solos no podrán cambiar la educación, pero si no cambian los docentes, difícilmente podrán lograrse transformaciones en ella. Los diferentes diagnósticos acerca de los mejores aprendizajes logrados por los estudiantes, y el incremento de la calidad de la educación, subrayan la importancia de los maestros como actores centrales en el proceso educativo" (González, 2008, p. 11).

El no tomar decididamente el compromiso de cambiar, causa dificultad en el momento que se evidencia el alcance de los objetivos de la educación inclusiva. Aunque, las guías a seguir por el MEN son prácticamente admirables por su contenido. La experiencia nos muestra que todavía hay mucho por hacer para reducir las desigualdades y obrar con equidad: hay carencias básicas de infraestructura en colegios y escuelas, utilización de términos ofensivos hacia estudiantes con NEE (anormales, mochitos, enfermitos, mancos y otros peyorativos más), pocas o nulas capacitaciones a docentes y administrativos sobre el tema, currículos y evaluaciones poco flexibles. Esta demanda de modernidad se revela, ocasionando deserción y repitencia por parte de los estudiantes, sentimientos de impotencia, ira y aflicción por parte de sus padres, así como también inconformismo por parte de algunos profesores. Pero no es el hecho de criticar simplemente, hay que proponer, y no basta con reconocerlo, hay que actuar, aunque esta posición implique un sendero de tristes recaídas pero también de muchísimos avances positivos colmados de satisfacciones.

Pero, como la realidad es una construcción del sujeto, y en esta realidad nos enfrentamos a la insensibilidad, miedos, facilismo e individualismo; todo lo que genere cambio o transformación causa inseguridad y ansiedad, asociadas a menudo a prejuicios e ideas que trasmitidas de boca en boca, pueden dar una imagen confusa de la educación Inclusiva, haciendo que los procesos de atención a la diversidad se convierta en un riesgo para atender la educación para todos con calidad.

Cabe anotar que como seres humanos con principios y valores, hemos ido perdiendo el valor de la empatía, la responsabilidad y la ética; cualidades necesarias para atender, guiar u orientar a nuestros estudiantes, más aún a estudiantes que requieren de nuestro profesionalismo y de pensamiento abierto hacia el cambio para que hagan historia y de alguna manera, de acuerdo a sus posibilidades contribuyan al desarrollo de nuestro país. 
Es necesario tener presente que en nuestro sistema educativo se vive un proceso muy complejo, que implica cambios radicales en el ámbito actitudinal, conceptual, metodológico y organizacional con la finalidad de implementar o establecer otras normas o modelos de intervención en las instituciones educativas en los niveles de prescolar, básica y media, para que tengan un perfil que favorezca la inclusión de personas con NEE. La dificultad de este proceso, entre otras ya mencionadas, está en la consolidación principalmente de los conceptos básicos que han de permitir la sensibilización, concientización y comprensión de la comunidad educativa y de la sociedad en general, con base en el principio de una educación para la diversidad.

Paulo Freire (1973) considera que los hombres tienen que tomar sentido de su propia existencia para poder ser personas, esa toma de conciencia supone capacidad de contextualizar su existencia y la de sus semejantes, este paso genera concienciación y radicalismo que sitúa y adapta al ser humano a la realidad. Hoy más que nunca, es urgente asumir el reto de aprender a vivir en un mundo globalizado, comprender la importancia de sentirse ciudadano del mundo y reconocerse como un sujeto activo y protagonista del progreso social. Desde esta perspectiva, la educación se convierte en una posibilidad para la formación integral del ser humano, contribuyendo al desarrollo de estrategias para aprender a aprender y mejorar las condiciones sociales, culturales, económicas y políticas del contexto actual.

Partiendo de la cotidianidad de los estudiantes que de forma permanente y continua asisten a un establecimiento educativo, es oportuno volver la mirada hacia la preparación del docente quién es uno de los responsables de la educación del ciudadano(a); Además de impartir sus saberes en diferentes disciplinas (matemáticas, español, ciencias naturales, sociales, inglés, informática, entre otras) debe buscar respuestas que contribuyan a cristalizar sociedades más justas. No se trata de formar un docente poseedor de gran cantidad de conocimientos y transmisor de información, cuyo objetivo en la mayoría de los casos es alcanzar a cumplir con su programa de instrucción durante el año lectivo sino de propiciar el desarrollo de capacidades, actitudes y aptitudes para el aprendizaje permanente.

Estamos viviendo en una época muy acelerada, en donde tenemos que responder a las exigencias de esta misma, por lo cual no dejo de lado la importancia de saberes de todas las áreas que nos ofrece el sistema educativo, sin embargo urge la necesidad de detectar lo que no muestran nuestros estudiantes, lo invisible; es ver en ellos sus potencialidades para explotarlas y empezarlos a orientar para que lo utilicen en el beneficio y desarrollo de la humanidad.

Educar también es formar comportamientos y no simplemente dar conocimientos. Esos principios y valores crean en el estudiante un estado de conciencia que le permite detectar lo que está bien o mal hecho, de igual manera le determina sí actúa o no frente a una situación que puede generar malestar, tanto para él mismo como para la sociedad, de ahí que la ética y vocación del docente juega una papel muy importante en el acto de educar, es pertinente tener presente que el estudiante, una de las primeras personas que admira o tiene como ejemplo a seguir es el docente; en consecuencia "lo más grave que le pude pasar al país, es tener a los maestros aburridos y desmotivados" (Linares, 2004, 37).

\section{Desesperanza aprendida}

La indefensión aprendida o adquirida (learned helplessness) es una condición psicológica en la que un sujeto aprende a creer que está indefenso, que no tiene ningún control sobre la situación en la que se encuentra y que cualquier cosa que haga es inútil. Como resultado, la persona permanece pasiva frente a una situación 
dolorosa o dañina, incluso cuando dispone de la posibilidad real de cambiar estas circunstancias.

"La indefensión aprendida fue postulada por el psicólogo Martin Seligman, quien, exponía a dos perros, encerrados en grandes jaulas, a descargas eléctricas ocasionales. Uno de los animales tenía la posibilidad de accionar una palanca con el hocico para detener esa descarga, mientras el otro animal no tenía medios para hacerlo. El tiempo de la descarga era igual para ambos, ya que la recibían en el mismo momento, y cuando el primer perro cortaba la electricidad, el otro también dejaba de recibirla. En cualquier caso, el efecto psicológico en ambos animales era muy distinto; mientras el primero mostraba un comportamiento y un ánimo normal, el otro permanecía quieto, lastimoso y asustado, con lo que la importancia de la sensación de control en el estado de ánimo parecía demostrada. Incluso cuando la situación cambiaba para el segundo animal, y ya sí podía controlar las descargas, era incapaz de darse cuenta y seguía recibiendo descargas sin intentar nada para evitarlo" (Ahumada, 2008, p. 23)

Pero ¿por qué este tecnicismo moderno es aplicado a los docentes, si ellos son seres humanos transformadores positivos de vida? Pues bien, para explicarlo tomaremos como muestra 2 preguntas realizadas a diferentes docentes: 1 . ¿Cree Usted pertinente la asistencia de estudiantes con NEE en las aulas regulares? D.1. "No, su educación debe estar en aulas especiales".D.2. "No ellos deben ser atendidos por personas que han sido capacitadas para tal fin".D.3. "No, porque para esto es necesario tener aulas, material pedagógico y personas especializadas para lograr una buena atención y desarrollo" D.5. "No, porque no se ofrece una garantía de asistencia integral. Como por ejemplo: planta física adecuada, personal humano capacitado, material didáctico requerido para determinadas discapacidades".D.6. "Sí siempre y cuando estas aulas estén debidamente atendidas por un equipo idóneo para la atención de los estudiantes con NEE".D.9. "Yo creo que depende del problema, hay casos que como docentes podemos ayudar, por ejemplo niños síndrome Down, aunque en un colegio como este no, ya que en niño especial se queda siempre atrás, depende de la discapacidad que tenga, porque hay una más leves que otras".

Una aportación sumamente interesante en el campo de la motivación es la relacionada por Seligman (1975), y su concepto de desesperanza (indefensión) aprendida que ocasiona déficits motivacionales, cognoscitivos y afectivos. Consiste en percibir que no importa lo que uno haga, ya que esta condenado a fracasar (Maier, Seligman y Solomon, 1969). La desesperanza (indefensión) aprendida puede aparecer a través de las prácticas de crianza (Hodoka, Frincham, 1995), pero también por el uso de formas impredecibles e inconscientes de reforzamiento y castigos por parte del profesorado. Aterrizando esta teoría a los docentes se encuentra que tienden a concebir las prácticas pedagógicas de forma negativa hacia los estudiantes con $\mathrm{NEE}$, considerando que son "una piedrita en el zapato", para desarrollar su habitual proceso de enseñanza.

Se limitan a observar su incapacidad y se niegan a descubrir sus habilidades y potencialidades, tienden a llevar a cabo adaptaciones que requieren poco esfuerzo y dedicación. La falta de tiempo, formación y recursos son algunos factores que pueden estar detrás de estos resultados, pero que lamentablemente daña y estanca al sujeto que esta en formación.

Ante la pregunta 2 . ¿ Ha tenido la oportunidad o experiencia de contar con un estudiante con NEE en su aula? ¿Cómo Usted se ha sentido? Cuando la experiencia es satisfactoria, la labor de la enseñanza puede constituir una fuente 
eminente de auto-realización personal, dando sentido a la vida profesional como pedagogos, pero sí esta en sentido contrario y al valorar la influencia de los efectos producidos por los problemas profesionales, esto ocasiona desánimo y pesimismo, consecuencia causada por el desconcierto al enfrentarse con los problemas reales que plantea la enseñanza contrapuesta a los ideales objetivos de una educación con calidad para todos.

D.1. "Sí, tenía una niña con retraso mental y me sentía muy mal, incapaz, solo dejaba que ella pintara, no hacia nada más"D.5. "Sí, Me sentí mal, incapaz de dar una verdadera orientación que el estudiante necesitaba, no tenia la preparación requerida para atender tal necesidad y por tal motivo lo aislaba" D.8. Lamentablemente nos encontramos ante una desmotivación, bajo nivel de satisfacción, desgano para el cumplimiento del compromiso laboral y social. Pareciese que en ocasiones se olvida que ser docente es una actividad de relación interpersonal, por cuanto se trabaja con seres humanos, aunado se presenta la falta de formación que sustenta la excusa "no estoy preparado para esto", pretendiendo ser la excusa para evadir la responsabilidad de brindar una educación de calidad.

Las percepciones y actitudes de los docentes puestos en consideración, expresadas en indiferencia, rechazo, segregación, negativismo, desmotivación hacia los estudiantes con NEE, se ha constituido en una de las causas para que sean excluidos y esto a su vez, puede ocasionar una desesperanza aprendida para ellos. Woolffolk (1999), plantea que "la desesperanza aprendida es un peligro muy serio, en especial en los estudiantes con problemas de aprendizaje, historia de fracaso escolar, y para aquellos que son víctimas de discriminación por diversos motivos género, clase social"

Esta actividad profesional encierra en sí unas dificultades cuya presencia es prácticamente permanente, a las cuales el profesor debe hacer frente de una manera coherente, aceptándolas y buscando soluciones de superación, para conseguir de esta forma, que la educación lleve consigo auto-realización. Con esto no se pretende dar una visión negativa y problemática de la educación, ni mucho menos negar ni ocultar sus inconvenientes, sino plantear la situación para un mejor desafío y conocimiento. "Se requieren cambios trascendentales en el ser maestro, es decir, docentes que se permitan pensar su acto educativo con sensibilidad humana, con solidaridad, ética y con un profundo amor pedagógico, en otras palabras un maestro posibilitador y no un maestro que genere barreras" (Lopera, 2004, p. 154).

\section{De la desesperanza a la esperanza aprendida}

"Todo acto educativo encierra una opción democrática o autoritaria, asumiendo en tal sentido una opción política "toda educación es un acto político, no solo por el ejercicio formativo en sí mismo, sino por sus consecuencias" (MEN, 1998,15). "De allí que se haga necesario reconocer el propósito fundamental de toda educación, cual es el de preparar para el mundo de la vida. Donde se encuentra el individuo que debe cuidarse a sí mismo y a la colectividad a la que se debe cuidar conjuntamente. Esta posibilidad conlleva a analizar el acumulado cultural que se reproduce y produce en toda acción educativa y las maneras como estas son enseñadas y aprendidas" (Díaz, 2003, p. 56).

La educación es parte fundamental de la formación del individuo, como dirección de humanidad e instrucción de conocimientos tiene un papel o una tarea muy importante en lo que se refiere al éxito o frustración de este, por la cual, urge la necesidad de dar respuestas a los desafíos del siglo XXI, cultivando al sujeto y preparándolo para la vida y la sociedad con saberes diversos y formas 
de sentir humanizadas que desarrollan una cultura capaz de enfrentar los retos que trae la globalización. Por ello, es de gran importancia hacer que nuestros estudiantes adopten un papel activo dentro del aula, como críticos principales, de sus docentes, de los problemas y las soluciones sociales actuales.

Pero lo anteriormente expuesto no se consigue únicamente cuando se plasma en un papel, lo difícil es llevar a la práctica lo estipulado en documentos o normatividades; es por esta razón que los formadores de niños, niñas y jóvenes necesitan reconocer que se educa sobre una realidad y se debe por tanto responsabilizarse de ella. Se puede trascender la situación de diversidad con el compromiso a trabajar por su transformación; una transformación que tome como como base el respeto hacia la diferencia, que desarrolle o potencialice al máximo habilidades y capacidades físicas, emocionales, intelectuales, sociales y personales de su ser como estudiante. En este caso, este pensamiento introduce la necesidad de un tipo de conocimiento constructivo e interpretativo que permita ir más allá de los límites de las representaciones conscientes. "El sujeto es sujeto del pensamiento, pero no de un pensamiento comprendido de forma exclusiva en su condición cognitiva, sino de pensamiento como proceso de sentido, que actúa solo a través de situaciones y contenidos que implican la emocionalidad de este" (González, 2008, p. 4).

Es necesario ser conscientes que como docentes a diario nos vemos avocados a crear en el aula un espacio motivador de conocimiento compartido, un proceso de comunicación abierta que concrete una intervención diferenciada desde los saberes previos y el uso de estrategias pedagógicas que prioricen el intercambio grupal e interpersonal y que favorezcan la participación de todos los estudiantes. La población estudiantil vive dentro de un escenario totalmente diverso, multicultural, como en el caso de las instituciones a las cuales se les realizó las entrevistas, y algunos de ellos, nos expresaban que tuvieron la posibilidad de tener en su clase estudiantes con Necesidades Educativas Especiales, después que los docentes rompieron esas barreras que poseían, empezaron a reconocerse y surgieron diversas formas de prácticas pedagógicas en las que los estudiantes aprendían a tomar posturas críticas a debatir y esforzarse colectivamente para convertirse en sujetos activos dentro del aula, ya que no sólo se trata que la población multicultural esté dentro de las aulas, sino que formen parte activa dentro de las mismas, comprometiéndose con el mundo, de manera que puedan plantearse cuestiones sin temor a recibir castigo alguno.

"La tendencia a retirarse de los espacios públicos hacia islas de uniformidad se convierte, con el tiempo, en el mayor obstáculo a la convivencia con la diferencia” (Bauman, 2006, p. 105), así mismo, "cuanto mayor es el temor, y más grande la distancia que se impone a aquellos a los que se teme, menores son las probabilidades de que se logre comprenderlos, aceptarlos" (Santiago, 2006, 10). Tal vez, sean estos, uno de los tantos argumentos con los cuales se puede justificar la impotencia de los docentes ante la formación de estudiantes que presentan Necesidades Educativas Especiales, es decir, encontrar la razón para el estado de pérdida de la motivación, la negligencia y la renuncia a posibilidades de mejorar la situación de "enseñabilidad" (Flórez, 1994).

Sí el docente tan sólo se diera la oportunidad de interactuar con un niño, niña o joven en condición de discapacidad, sin que fuera una camisa de fuerza obligada por el estado, sino que por el contrario lo recibiera como un reto positivo para transformar vidas, muy seguramente conocería la grandeza de la misión de educar y la magnitud de la responsabilidad social de la misma. Ciertamente, los docentes requieren ser conscientes que la educación Inclusiva trae el sello de compromiso, responsabilidad y amor, como elementos para anticipar respuestas a nuevos de- 
safíos y generar alternativas de solución, pues las innovaciones educativas se presentan como un espacio crucial para la educación en y desde la diversidad.

En este tema de la educación inclusiva todo no es desesperanza aprendida, hay excepciones que hacen la diferencia, que han logrado dar respuesta a las Necesidades particulares de los estudiantes, desde sus propias iniciativas pedagógicas y didácticas, como por ejemplo; la experiencia evocada por los docente D.6, y D7, quienes respondieron: "en mi caso, tengo la dicha de compartir con dos niños ciegos y para esto, se tuvo que hacer unas modificaciones curriculares y solicité apoyo a la Unidad de Atención Integral"; "me sentí inicialmente preocupada, pero al poco tiempo sentí la satisfacción de haber participado y aportado al proceso de inclusión", "cuento con un nivel básico de formación pero se hace necesaria una formación profesional y sistemática en este campo". D.7 "busqué ayuda, me sentía incapaz, así que busqué ayuda en el INSOR y en el INALE y poco a poco seguí adelante con ellas y me sentí muy orgullosa, yo creo que ha sido una de las experiencias más maravillosas en todo mi recorrido académico"

Lo anterior demuestra que es factible hablar de educación inclusiva de calidad, para ello, hay que re-plantear la actitud que se tiene frente a la verdadera vocación de ser docentes. Cabe anotar que en cómo se afronta la desesperanza, la experiencia de vida es un elemento fundamental, pues dependiendo de cómo se viva, y de la manera de relacionarnos con los demás que se provoca un encadenamiento de experiencias significativas, maravillosas y fortificantes que permite alcanzar plenitud y paz. La alegría que se siente ver culminar un objetivo o saber que se está contribuyendo eficazmente en la formación de autonomía y funcionalidad de un estudiante con NEE da cuenta que no equivocaste la elección de tu profesión y este factor gratificante concede motivación para seguir fortaleciendo el proceso educativo y por ende ser partícipe de dignificar vida; pues sentir confianza en sí mismos, acrecienta la motivación de todo niño, niña o joven que esté en proceso de formación.

Esta nueva visión de cómo debe ser la educación en la reforma del nuevo milenio tiene como meta educar a todo los estudiantes como seres diferentes, únicos y con una multiplicidad de talentos, necesidades, intereses y aspiraciones (Santana 2005). Por lo tanto, se hace necesario trabajar en una educación diferenciada, que brinde a cada individuo la oportunidad de exponerse a experiencias de aprendizaje en las que se tome en consideración sus intereses, capacidades y fortalezas. La función del maestro será dirigir a sus niños hacia el desarrollo de valores de aprecio, respeto y tolerancia hacia la diversidad.

\section{Conclusiones}

Los resultados parecen demostrar que el profesorado en algunos casos ha realizado prácticas inclusivas independientemente de la formación que ha recibido o de los recursos de que dispone en la Institución Educativa, con resultados negativos, conducidos por la desesperanza aprendida, logrando visualizar en ellos, desmotivación, impotencia, exclusión y la predisposición que generan entre ellos al momento de orientar su saber pedagógico a estudiantes que presentan Necesidades Educativas Especiales.

Contrario a esta experiencia también se evidencian circunstancias positivas que enaltecen el quehacer pedagógico, causando gratificación tanto a nivel personal como profesional de las personas que han estado sumergidas en esta labor tan dispendiosa; comentarios como: "me sentí muy orgullosa, yo creo que ha sido una de las experiencias más maravillosas en todo mi recorrido académico" y "sentí la satisfacción de haber participado y aportado al proceso de inclusión", contagian el espíritu inclusor de docentes comprometidos, generando una esperanza aprendida. 
Por otra parte el desconocimiento de los docentes sobre temas relacionados con la atención a la diversidad, aparece como otro factor importante en sus actitudes y percepciones, como ejemplo a rescatar, teniendo en cuenta que estamos en el siglo XXI y supuestamente estamos en la vanguardia y sujetos al cambio, encontramos expresiones como: "no hay que rechazarlos, siempre y cuando vayan a un aula especial”. ¡Que tristeza, da esta respuesta!, puesto que somos formadores de seres humanos y sobre cada docente recae la responsabilidad de brindar una educación integral, equitativa e igualitaria.

El rechazo social, la etiqueta que se le da a una persona por considerarla "diferente", generalmente se asocia con imágenes o preceptos mentales negativos, aunque muchos de ellos, parecen tolerar este flagelo generalizado desafortunadamente por sus semejantes, convirtiéndose en un reto poderlas resistir, y transformarlas en fuente de reflexión y sabiduría.

Sí bien es cierto que hay un porcentaje de docentes que cuentan con muchas ansias para mejorar su praxis cotidiana y vocación para ser transformadores de vida, hay también muchos en ejercicio que no han adquirido o se han negado a una formación pensada para la escuela del siglo XXI, y menos aún se ha considerado el hecho de que la sociedad está siendo objeto de muchos cambios, donde sin duda alguna, la formación permanente resulta indispensable para el profesorado de todos los niveles educativos.

La creciente diversidad de las y los estudiantes en los centros educativos enriquecida por la inclusión de estudiantes con NEE dentro de las aulas regulares, ha evidenciado una serie de necesidades, actitudes y percepciones tanto positivas como negativas en los profesionales de la docencia que conduce a replantear la verdadera vocación del docente, no solo como facilitador de conocimientos y procesos de enseñanza - aprendizaje, sino como orientadores, capaces de asumir un papel relevante en el camino hacia este modelo inclusivo que persigue y necesita la sociedad actual.

Sin embargo, la búsqueda de una educación de calidad para todas las niñas, niños y jóvenes, independientemente de cuáles sean sus circunstancias o características debe ser el propósito de todas las instituciones educativas y, por ende, de las personas que trabajan en ella. Es decir, trabajar para humanizarse y humanizar. Es así, como en el ejercicio de la labor docente, se evidencian innumerables características individuales que hacen la diferencia en el quehacer pedagógico.

Sumado a lo anterior, se evidencia la necesidad de construir una comprensión transformadora acerca del desempeño profesional (que dicho agente ostenta en los centros educativos) y las dimensiones que este nuevo rol esta marcando, dimensiones generadas por los cambios en la sociedad y en los centros educativos en particular, esto con la finalidad de no dar respuestas educativas excluyentes.

Es sumamente dispendioso desde el punto de vista moral y psicológico ser rechazado por simplemente ser, como también desarrollarse por vivir experiencias. Es muy costoso el precio que se paga por el insulto, la indiferencia, la simple no aceptación o reconocimiento de la diversidad. Todos los seres humanos, aunque sea difícil de reconocer, sólo somos iguales al mismo tiempo que somos diferentes.

\section{Recomendaciones}

El proceso de enseñanza-aprendizaje a desarrollar en la escuela, facilita la innovación, si se tiene una nueva mentalidad en todos los implicados en el proceso educativo. Sin obviar las características individuales de cada estudiante, no se pretende en ningún caso ignorar los posibles necesidades de los alumnos provenientes de otras culturas o de niveles sociales determinados, al contrario ofrecerles a partir de sus capacidades y competencias una 
respuesta educativa adaptada y eficaz a sus necesidades.

Realizar procesos de capacitación y auto-capacitación en torno a la atención de la población diversa, con el fin de replantear paradigmas negativos que en ocasiones la misma sociedad nos impregna, además de generar, una actitud abierta a la diversidad, construyendo de forma positiva la aplicación de estrategia didácticas

Adaptar la escuela a las necesidades de sus estudiantes y no posibilitar el proceso inverso; adecuar la educación a la realidad actual que vivimos, no desde la selección y/o compensación para alcanzar una uniformidad y homogeneización utó- pica e imposible, sino desde la atención y el respeto a la diversidad de todos los alumnos, sin excepciones, para lograr una verdadera igualdad de oportunidades.

Los apoyos pedagógicos y didácticos en una escuela que pretende dar una respuesta a la diversidad de los estudiantes desde lo común y lo ordinario a través de las adaptaciones del currículum, precisa de una organización que contemple un modelo curricular en el cual, los apoyos sean componentes del mismo; esto implica una definición concreta de los roles y de las funciones a desempeñar por los diferentes profesionales que actúan en la escuela, según los criterios expuestos con anterioridad.

\section{Bibliografía}

\section{Fuentaes}

Ahumada. Víctor. (2008). El experimento de Seligman: indefensión aprendida. En: Periódico Zócalo. Sección de opinión. 26 Agosto de 2008. (Recuperado en agosto de 2013).

Bauman, Zigmund. (2006). Vida líquida, Paidós Ibérica. Disponible en: http://www.zocalo. com.mx/seccion/opinion-articulo/el-experimento-de-eligman-indefension-aprendida. (Recuperado en octubre de 2012).

Blasquez Santana, Felix. (2005). Administración Educativa Inclusiva: Retos para los sistemas educativos en el nuevo milenio. San Juan: Publicaciones Puerto Riqueñas.

Bruner, Jerome y Haste, H. (1990). La elaboración del sentido: la construcción del mundo por el niño. Barcelona: Editorial Paidós.

Cedeño, Fulvia. (2005). Colombia, hacia la educación inclusiva de calidad. MEN. Bogotá: Subdirección de Poblaciones República de Colombia.

Cepal-Eclac. Vulnerabilidad Sociodemográfica: viejos y nuevos riesgos para comunidades, hogares y personas. Brasilia: ONU, LC/R.2086, 22 abril, 2002.

Díaz, Álvaro (2003). Una discreta diferenciación entre la política y lo político y su incidencia sobre la educación en cuanto a la socialización política. Reflexión política. Mes junio.
Año 2003. Número 9. Universidad Autónoma de Bucaramanga. Colombia. pp. 49-58.

Flórez, Ochoa, Rafael (1994). Hacia una pedagogía del conocimiento. Bogotá: Editorial McGraw-Hill.

Freire, Paulo. (1973). La Educación como Práctica de la libertad. Buenos Aires: Siglo XXI.

Freire, Paulo. (1999). La Pedagogía de la Esperanza un re-encuentro con la pedagogía del oprimido. Editorial Siglo XXI. Disponible en: http://issuu.com/zinias/docs/ pedagog_a_de_la_esperanza. (Recuperado en julio 18 de 2011$)$.

Giroux, Henry. (1.992). La Pedagogía de Frontera y la Política del Postmodernismo. En: Revista Intríngulis № 6. Sept - Dic. 1992. pp. 33-47. Disponible en: http://www.pedagogiasocial.cl/DOCS/pdf/pedagogia\%20 de\%20la\%20frontera.pdf. (Recuperado en agosto 12 de 2012).

Glaser, B. y Strauss, A. (1967). The discovery of grounded theory: strategies for qualitative research, New York: Editorial Aldine de Grunter.

Gobernación del Cauca. Dirección Departamental de salud, Despacho de la primera dama y Red de iniciativas de personas discapacitadas COMUNITAR. (1999). "Discapacidad, minusvalía y deficiencia; Alertas y procesos en el Departamento del Cauca". 
González González, Miguel Alberto. (2014). Miedos y olvidos pedagógicos. Rosario: Homosapiens.

González Lara, Mireya. (2008). Paradojas en la formación docente. Elementos para avanzar en su reflexión y planteamiento de propuestas. Bogotá, D.C., Colombia.

Isaza de Gil, Gloria. (2011). Antecedentes de los elementos epistemológicos que han dado cuenta de los procesos pedagógicos. Manizales: Universidad de Manizales. CEDUM. Disponible en: http:// cedum.umanizales.edu.co/epistemologia/ epistemologia_y_pedagogia/material_apoyo/elementos_epistemologicos.pdf. (Recuperado en agosto de 2012).

Linares, Jorge Duque. (2004). La misión de educar, Cuarta Edición. Bogotá: Editorial Eduque.

Lopera, Jorge. (2004). El maestro ante la diversidad. En: Modulo 1 de capacitación, Medellín, Diversidad y Educación. Capítulo 1. Educación para personas con limitaciones o capacidades excepcionales. Santafé de Bogotá: Editorial Ecoe.

Maier. S.F., Seligman, M.E.P., and Solomon, R.L. (1969). Pavlovian fear conditioning and learned helplessness. In Campbell, B.A., and Church, R.M. (Eds.), Punishment. New York: Appleton-Century-Crofts.

Ministerio de Educación Nacional. (1994). Ley 115 de Febrero 8 de 1994. Titulo III.

Ministerio de Educación Nacional. (1997). Una escuela con alas. Decreto 2082 de 1996, Discapacidad, minusvalía y deficiencia.

Ministerio de Educación Nacional. (2009). Decreto 366 de Febrero 9 de 2009. Bogotá: Imprenta Nacional.

Patiño Giraldo, Luz Helena. (2011). La atención a la diversidad en el contexto del aula de clase. Modulo Alternativas pedagógicas. Manizales: Universidad de Manizales.

Sandoval, Casilimas. (1997). Investigación cualitativa. Módulo 4. Programa de especialización en teoría, métodos y técnicas de investigación social. Medellín: Universidad de Antioquía.

Santiago, Gustavo. (2006). De una modernidad a otra. Disponible en: http://familiaydiversi- dad.blogspot.com/2007/05/de-una-modernidad-otra-man.html. (Recuperado en Julio 21 de 2012)

Seligman, Martin. (1975). Helplessness: On Depression, Development, and Death. San Francisco: W.H. Freeman.

Woolfolk, Anita. (1999). Psicología Educativa. (7 edición). México: Prentice Hall Hispanoamerica, S.A.

Zemelman, Hugo. (1998). Sujeto: existencia y potencia. Barcelona: Anthropos/UNAM.

\section{Referencias}

CEPAL. (2009). Panorama Social de América Latina 2002-2003. México: Editorial Cepal

Constitución Política de Colombia (1991). Título II, de los derechos, las garantías y los deberes, artículo 67. Santafé de Bogotá: Editorial Magisterio.

De la Cuesta, B. (1998). Taller de investigación cualitativa. En: Memorias del seminario taller de investigación cualitativa, Universidad de Caldas. Manizales: Facultad de ciencias para la salud, universidad de Caldas.

González González, Miguel Alberto. (1998). Los amores prohibidos de Kalkan. Pereira: Editorial Papiro.

Grisales, María Carmenza. (2011). El reconocimiento de la diversidad como valor y derecho. Maestría en Educación desde la Diversidad. Manizales Colombia: CEDUM.

Guarín Jurado, Germán. (2011). Modernidad Positiva. Modernidad Crítica. Módulo Modernidad Crítica: fundamentos epistémicometodológicos. Manizales, Colombia. Universidad de Manizales.

Hodoka, A., Fincham, F.D. (1995). Origins of children's helpless and mastery achievement patterns in the family. Journal Educational Psichology, 87, 375 - 385.

Ministerio de Educación Nacional. (1998). Constitución Política y Democracia. Lineamientos curriculares. Santafé de Bogotá: Cooperativa Editorial Magisterio.

Strauss, Anselm. (1970). Descubriendo nuevas teorías de teorías previas. En: T shibutani (comp): Naturaleza humana y comportamiento colectivo. Ensayos en honor a helbert blumer, Nueva Jersey: Prentice-Hall. 